\title{
On Structuralism's Multiple Paths through Spacetime Theories
}

\section{Abstract (WORD COUNT: 121)}

This essay examines the underdetermination problem that plagues structuralist approaches to spacetime theories, with special emphasis placed on the epistemic brands of structuralism, whether of the scientific realist variety or not. Recent non-realist structuralist accounts, by Friedman and van Fraassen, have touted the fact that different structures can accommodate the same evidence as a virtue vis-à-vis their realist counterparts; but, as will be argued, these claims gain little traction against a properly constructed liberal version of epistemic structural realism. Overall, a broad construal of spacetime theories along epistemic structural realist lines will be defended which draws upon both Friedman's earlier work and the convergence of approximate structure over theory change, but which also challenges various claims of the ontic structural realists. 
The contemporary revival of structuralism in the philosophy of science can be traced to a host of early twentieth century structuralist approaches, principally, the philosophical speculation on the epistemology and ontology of mathematical physics put forward by Poincaré, Eddington, Weyl, Russell, and Cassirer, to name just a few. Given the structuralist's fixation on the mathematical and/or empirical structure of theories, as opposed to, say, the underlying theoretical entities alleged to bring about and sustain those structures, it is therefore not surprising that the underdetermination of structure quickly became a serious obstacle for the new program; e.g., Newman's critique of Russell (see endnote 10). That is, since more than one structure is liable to be consistent with the same evidence, a theory's structure is as underdetermined as its ontology (for, as is well known, several different sets of unobservable entities can be reconciled with the same observation basis). This essay will examine the current crop of structuralist conceptions of science as regards the underdetermination problem, focusing specifically on spacetime theories. In contrast to those interpretations inspired by quantum mechanics, the spacetimes of classical gravitation theories have generally received less attention from structuralists, even though they more readily disclose the tensions between the competing ontic and epistemic orientations.

In section 1, these rival strains of structural realism, ontological and epistemological, will be evaluated in the light of the underdetermination problem in spacetime theories, for the difficulty is manifest by these approaches in divergent ways. After demonstrating, in section 2, the similarities between epistemic structural realism, early twentieth century structuralism, and Michael Friedman's idea of the relativized a priori, the role that underdetermination plays in his assessment, and, to a lesser extent, in van Fraassen's, will 
be the major concern of sections 3 . This section will also provide the details for an epistemic brand of structural realism that can account for the various underdetermination problems surveyed in earlier sections. Finally, a sophisticated form of ontic structural realism will be critiqued in section 4 .

Overall, the main theme of our investigation is that a properly construed broad or liberal conception of epistemic structural realism is best equipped to handle the underdetermination problem, or, put differently, that all other structuralist approaches must fall back on conceptual resources that are strikingly similar to the liberal brand of epistemic structuralism defended in this essay. Consequently, the embrace of "multiple paths" in scientific theorizing is not inconsistent with epistemic structural realism, but it does draw our attention to the convergence and constraints manifest in scientific theorizing, as well as Friedman's own early defense of realism. These last ingredients in the structural realist account have likewise received scant consideration, but are central to our goal of establishing the viability of epistemic structural realism for spacetime theories.

\section{Structural Realism and Underdetermination in the Spacetime Context.}

This section will not only explore the differences between OSR and a liberal brand of ESR, but much of the discussion will center on various defenses offered to counter potential objections raised by an OSR theorist against this unique form of ESR (in sections 1.2 and 1.3).

1.1. OSR and Liberal ESR. As a departure from a straightforward realism involving theoretical entities, Structural Realism (SR) holds that what is preserved in successive 
theory change is the abstract mathematical or structural content of a scientific theory. There are two principle virtues associated with SR. First, SR can explain the progressive empirical success of scientific theorizing, and thereby accommodates the "no miracles" argument, since the structures present in our best scientific theories pertain to, or capture in some manner, the "real" world (i.e., the success of science would be miracle without some realist commitment). Yet, secondly, SR avoids the "pessimistic meta-induction" (that plagues standard scientific realism) since it does not make an ontological commitment to the theoretical entities actually utilized by specific theories. Worrall's well-known example involves the progression in nineteenth century optics from Fresnel's elastic solid ether to Maxwell's electromagnetic field: “Fresnel's equations are taken over completely intact into the superseding theory [Maxwell's] — reappearing there newly interpreted but, as mathematical equations, entirely unchanged" (Worrall 1989, 120). What is less well-known, however, is Worrall's comment that this example is "unrepresentative", and that "the more common pattern is that the old equations reappear as limiting cases of the new-that is, the old and new equations are strictly inconsistent, but the new tend to the old as some quantity tends to some limit" (120, original emphasis). The case that is cited to establish this point is rather telling for the would-be spacetime structural realist, as will be disclosed more fully in section 2: "Einstein's equations undeniably go over to Newton's in certain limiting special cases. In this sense, there is 'approximate continuity' of structure in this case" (121). Inspired by Poincaré, Worrall's formulation of SR has become the basis of a view dubbed epistemic structural realism (ESR) in the literature, for it regards structure from a purely epistemological perspective, such that the mathematical structures that turn up in our best scientific 
theories do not, or may not, provide any information on the actual ontology of entities and processes that underlie the observed structural relationships. In short, ESR makes a realist commitment to the invariance of structure across scientific change, although the details of this complex story will only emerge after much discussion.

Ontic structural realism (OSR), in contrast, goes beyond a mere epistemological interpretation of structure; rather, structures $d o$ reveal facts or truths about the underlying ontology — and may, in fact, be the underlying ontology. OSR, as its name implies, thus includes mathematical structures and relations within its ontological assessment. ESR might seem closer to traditional scientific realism, accordingly, since an ESR theorist could regard the mathematical structures in our best theories as merely the epistemologically-assessable relations between the relata, i.e., the entities, such that only the entities are real (and not the relations themselves). But, this essay defends a more liberal form of ESR that is open to more options, namely, that the underlying ontology may include the relations alongside the relata in the same manner as OSR, although the precise ontological details are epistemologically inaccessible. There is no reason to deny this formulation of ESR, it should be added, and is in keeping with the skeptical orientation of ESR concerning our knowledge of nature's deep ontology. Henceforth, all references to ESR will take this more liberal or broad form; i.e., the underlying ontology can include only relata, only relations, or both relations and relata. The "no miracles" argument, in effect, would seem to constitute the main "realist" commitment of liberal ESR, for it holds that the approximate continuity of mathematical structure manifest over the history of any one science is not an accident, but is due to the "constraints" that reality imposes on our scientific endeavors (see Brading and Landry 2004 for a similar 
construal of ESR, and section 3).i Of course, all of the other forms of structuralism explored in this essay, with the likely exception of van Fraassen's, would also agree with the claim that nature's constraints are non-accidental features of our best theories.

As recounted in French and Rickles (2006), Cassirer and Eddington's reflections on the group structure of General Relativity (GR) and quantum mechanics (QM) motivates OSR, since the structural role that, say, quantum particles assume within the larger mathematical structure can allow a straightforward ontological reading, such that the particles no longer retain their autonomous, individual status usually associated with scientific realism; instead, it is the group structure itself which directly represents the ontology. Transferred to the arena of the modern spacetime ontology debate, OSR seeks to handle the points of the spacetime manifold in an analogous fashion: the points are either a derived, secondary aspect of the primary ontological unit, in this case, the metric; or, the points and metric, as relata and relation, are construed as being ontologically "on a par", such that neither is more basic. Of course, these interpretations have, until recently, been understood as a form of sophisticated substantivalism (e.g., Hoefer 1996), or even sophisticated relationism (e.g., Dorato 2000), but French and Rickles (2006, 24) claim that they are closer in spirit to SR.

1.2. Entity Underdetermination. While French and Rickles' assertion has much merit, the one major drawback of identifying sophisticated forms of both substantivalism and relationism with OSR is that the main goal of Worrall's initial plan for SR - namely, to uphold the "no miracles" argument and defeat the pessimistic meta-induction-is thereby neglected or forsaken altogether; that is, sophisticated substantivalism never attempted to counter anti-realist worries, since it is a metaphysical interpretation of spacetime theories 
with different goals in mind. The contribution of Esfeld and Lam (2008) is a case in point, for it strives to apply OSR exclusively to spacetime theories, and is quite candid in its assessment of ESR. After rehashing SR's desire to defeat the pessimistic metainduction à la Worrall, and correctly noting that the cumulative progress employed by SR pertains to approximate structure $(2008,28)$, they conclude that the arguments that support the pessimistic meta-induction apply to "views about structure as well", and thus "structural realism as such does not rescue scientific realism" $(2008,29)$. Discarding Worrall's original intentions for SR, Esfeld and Lam conclude: "we pre-suppose scientific realism, but we do not intend to use structural realism in support of scientific realism" $(2008,29)$.

Yet, Esfeld and Lam's negative appraisal of the prospects for SR is much too hasty. ESR, for example, does try to locate an invariant within the historical progress of science: hypothetical entities will come and go, but the retention of mathematical structure, both as an approximation and a limiting case, remains fixed. What the OSR theorists have provided, in contrast, is yet another form of realism based on unobservable entities, but which this time incorporates various aspects of mathematical/conceptual structure alongside all of the usual drawbacks endemic to a scientific realism based on unobservable entities and their intrinsic properties (more on this below). Returning to Esfeld and Lam, they defend a moderate form of OSR within the context of GR, such that the points and metric/fibres are on a par ontologically (utilizing both the standard tensor and fibre bundle formalisms), but then concede in the last few lines of the essay that "in the framework of certain candidates for QG [quantum gravity], such as loop quantum gravity or the algebraic generalization of GR, there may be no reference anymore to 
space-time points" (44). Consequently, by folding OSR into sophisticated substantivalism, the advocates of OSR for spacetime theories have ceded the field, albeit unintentionally, to the anti-realist's arguments against all forms of SR (including their own form of OSR). The anti-realist will happily point out that, once again, the "entities" (in this case, spacetime points) that appear in our best currently accepted theory will be eventually replaced by a different set of entities in the successor theory; hence one should draw an anti-realist conclusion on the existence of all theoretical entities, whether those entities are viewed as distinct individuals (e.g., spacetime points, electrons) or those entities subsume the individuals into their larger structure (e.g., metric, fibre bundles, group structure in QM).

One should not misconstrue the intention of this last criticism, however. It is not simply the argument that spacetime points will likely be discarded in QG: even if one adopts an eliminativist form of OSR, which denies the reality of spacetime points for the metric/fibre structures of GR, it will still be the case that those mathematical structures will almost certainly be replaced by the different mathematical structures used in QG; e.g., replacing tensors or fibre bundles in GR for the more fundamental Hilbert space, or replacing the standard formalisms in both GR and QM for some deeper mathematical structure employed by an underlying successor theory (such that GR and QM are now the limiting cases of this deeper theory). In short, underdetermination is a much greater threat to OSR, which reifies structure to some degree, as opposed to ESR, which does not. "Reifies", in this context, is not meant to signify a sort of Platonic world view that literally posits mathematical entities as existing alongside the physical ones-rather, it is meant to signify that specific aspects or general characteristics of the mathematical 
structure of our best current theories, say, GR or QM, are taken to directly represent or actually correspond to the underlying ontology. For example, since the group structure in QM engenders a sort of holistic picture of particles, such that the individual particles cannot be separated from the field encoded in the group structure representation, most QM-inspired OSR theorists have drawn the lesson that the world's underlying ontology must be identically non-individualistic or holistic: e.g., "world-structure".ii The underdetermination that effects the ontic structural realist, consequently, is but an instance of the same underdetermination problem common to all brands of scientific realism that employ theoretical entities, a problem which we can dub "entity underdetermination".iii In short, while our best present-day spacetime theories support GR, as opposed to, e.g., a Machian alternative, it will likely be superseded by a more fundamental theory, such as one of the many competing QG hypotheses. Given this likely outcome (since GR is a classical gravitational field theory that is strictly inconsistent with quantum mechanics), GR will thus be seen as approximately true at large scales of space and time in the sense that its equations are a limiting case of the more fundamental theory and its different class of entities and mathematical structures - and this, of course, is exactly what ESR predicts, hence the rationale for ESR over OSR.

The same reasoning applies in the case of QM. While group structure is an entrenched component of modern particle physics, it is possible that a successor theory may come along that replaces group structure with a non-holistic mathematical or conceptual alternative — with a robust, non-eliminativist role for individual particles or other entities - thereby overturning the motivation for the holistic world-structure ontology: i.e., group structure would then be seen as a higher level aspect of the 
phenomena that is reducible to this more fundamental, non-holistic, individualspreserving structure of the hypothetical successor theory. As a result, the anti-realist's contention that "all entities are eventually replaced" will have been vindicated, since the world-structure ontology will have been called into question by the ascension of the nonholistic successor theory. There are a host of speculative theories in QG that would seem to raise this very challenge for $\mathrm{QM}$ and its standard formalism, prompted in no small part by the enduring obstacles faced by the more prominent QG theories, e.g., String theory and Loop Quantum Gravity. According to Butterfield and Isham, "[t]he idea here is that both classical general relativity and standard quantum theory emerge from a theory that looks very different from both.... presumably by not being a quantum theory, even in a broad sense- for example, in the sense of states giving amplitudes to the values of quantities, whose norms squared give probabilities" (Butterfield and Isham, 60). Some of these alternatives involve the use of quantum computational procedures modeled on quantum cellular automata, the latter being discrete, cell-like computational devices with local neighborhoods modeled on electronic circuits: "The probably best-known of these emergent quantum approaches goes back to 't Hooft, [who] proposes a deterministic, pregeometric, non-quantum substrate, which should possibly be modeled by something like cellular automata" (Hedrich 2009, 25). Other examples are addressed by Butterfield and Isham, who describe QG strategies that reject the real and complex numbers standard in group theoretic constructions of QM:

So, according to this line of thought, the use of real numbers (and similarly, complex numbers) in quantum theory in effect involves a prior assumption that space should be modeled as a continuum. If so, then the suggestion that standard spacetime concepts break down at the Planck length and time, and must be replaced by some discrete structure that only 'looks like' a differentiable manifold at large scales, 
means that we cannot expect to construct a theory of this discrete structure using standard quantum theory-with its real and complex numbers. (85)

Furthermore, if the OSR theorist were to insist that all of these potential successor theories still exemplify their world-structure ontology, then the very meaning of that ontology would appear to be strained beyond credibility. For instance, even granting that a flow of information connects the cellular automata, a series of local, discrete quantum computers seems much closer to the standard individuals-based conception of QM than the holistic "world-structure" ontology based on group structure. Likewise, the lawless, chaotic non-QM substrate, originally conceived by Wheeler as an early instance of a QG theory, would seem to be the very antithesis of a world-structure ontology (see, once again, Hedrich 2009, 25).

Of course, it could be the case that the OSR theorist is right, and that all successor theories will uphold the group structure in contemporary QM (or an equivalent holistic conception), such that the world-structure ontology is vindicated, contra anti-realism. The ESR theorist, on the other hand, takes the anti-realist challenge of the pessimistic metainduction as a motivating principle, and this prompts their skeptical approach to ontology. On this issue, ESR may thus be symptomatic of the more "anti-realist friendly" environment in spacetime theories, where different structural approaches abound, unlike QM and its seemingly ubiquitous, at least thus far, mathematics of group structure.

\subsection{Formalism Underdetermination. Returning to the analysis of spacetime theories}

and SR, there is another form of underdetermination implicit in Esfeld and Lam's comment on the possible overthrow of spacetime points. The reference to algebraic forms of GR raises the specter of a "formalism underdetermination", which can be seen as a variant of the entity underdetermination just divulged for OSR, although it is not 
necessarily tied to the inevitable succession of theories over time (e.g., GR replacing Newtonian Theory). In short, there are many competing mathematical formulations of any given spacetime theory, and, while some of these formulations may be better suited for an extension to the quantum realm, they need not be. For instance, Cartan showed that Newtonian Gravitation Theory could employ a mathematical treatment similar to that advanced in GR, such as the use of a non-flat connection and a geometrized gravitational potential, which is strictly inconsistent with the flat inertial structure of standard Newtonian theory (see, e.g., Pooley 2006).iv In the case of GR, there are a number of competing formalisms from which to choose: in addition to the typical tensors on manifold method, there are the twistor, Einstein algebra, and Dirac algebra formulations surveyed in Bain (2006).v This formalism underdetermination also afflicts ESR, of course, although we will postpone that discussion for now (see section 3).

Furthermore, in the context of QM, one cannot rule out the possibility that an alternative to the group structure formalism of QM may also be devised, such that this alternative formalism challenges the world-structure hypothesis by admitting, in some fashion, a non-holistic role for individual particles (i.e., a new approach that is without the drawbacks that are common for individuals-based interpretations of the standard QM formalism; see, e.g., French 1989, for individuals versus non-individuals interpretations of QM). The OSR advocate of the world-structure ontology could reply, here, that these alternative, individuals-based formalisms would simply be limiting cases of the holistic group structure formalism, but then the question remains open as to which formalism correctly singles out the underlying ontology. Maybe the new individuals-preserving 
formalism represents the actual ontology, with the group structure formalism constituting a mere limiting case of a non-holistic realm of individual quantum particles?

Returning to the general topic of formalism underdetermination in the context of spacetime theories, another response that the spacetime advocate of OSR might offer is that these alternative mathematical formulations, if they are truly grounded in an identical physical theory, are much more akin to different hierarchical arrangements of the same geometric component structures (manifold, metric, affine, conformal, etc.), rather than different geometrical structures altogether: e.g., conformal structure is basic for the twistor theorist, with the manifold and metric as derived structures, whereas manifold and metric structure is basic for the traditional tensor theorist, and conformal structure is derivative. As Bain aptly notes in his explanation of these basic/higher-level orderings, "what is real, the spacetime structuralist will claim, is the structure itself, and not the manner in which the alternative formalisms instantiate it" (2006, 64; see, also, Slowik 2005). Although this response is neutral as regards the OSR/ESR dispute, it would seem to be more appropriate coming from an ESR theorist, who takes an epistemological stance on mathematical structure. Moreover, how does an OSR theorist accept an ontology of spacetime structures per se, or in general, as opposed to a particular mathematical treatment of spacetime structures? That is, someone who sides with an ontology of "the geometry of shapes and angles" (conformal structure) or any of the other general structures in a spacetime theory, rather than accept an ontology tied to a specific mathematical theory and its formalism, say, twistor theory, would seem to be advocating a metaphysics of universals, and not particulars (with twistor theory being a particular instantiation of those universals). Would the OSR theorist happily accept this outcome, 
and sanction general geometric structures over particular instantiations of those structures? The world-structure ontology in QM would appear susceptible to the same problem as well, since a general non-individuals metaphysics is the basis of any mathematical instantiation of that ontology, such as group structure in QM. In essence, "a non-specific, general ontological structure" might be a contradiction in terms, and would appear to be much more appropriate if translated into "a non-specific, general epistemological structure that is grounded in a specific, non-general ontology" (i.e., ESR).

To recap, ESR has a more palatable account to offer, since it maintains a strict neutrality on the underlying ontology: that ontology might coincide with either traditional scientific realism, such that the mathematical structures are entirely phenomenal and are mere relations among old-fashioned physical entities (which is largely why Psillos 2004 finds less to fault in ESR); or ESR will ultimately coincide with a particular spacetime formulation of OSR, such as twistor theory—but it need not endorse an ontology of spacetime structures "in general", or world-structure.vi In other words, both the entity and formalism underdetermination problems are, for ESR, simply a result of our inability to empirically and theoretically determine the nature of the underlying ontology, but they do not, as in the case above, need to counter those underdetermination worries by saying that the underlying ontology really is just a loosely-defined collection of generalized geometric properties, or world-structure: consequently, the metaphysical implications of liberal ESR seem much less radical than for OSR.

In reply, the OSR theorist might insist that the underdetermination of structure in our best spacetime theories is merely descriptive or representational, such that there are many 
ways to characterize the world's structure. The use of correspondence principles that can connect and correlate different mathematical formulations of the phenomenon, say, classical physics and QM, would be an instance of this approach (see, e.g., Saunders 1993). Of course, the OSR theorist can perfectly endorse these maneuvers, and they are certainly not contradictory. Yet, the lesson to be gleaned from these competing hierarchical compositions of component geometric structures, as well as from the use of correspondence principles in QM, is that one should not accept the literal truth of mathematical formulations - but, this good advice would seem to contradict what OSR counsels, since its world-structure ontology is indeed directly based on an important feature of the group structure formalism in QM (as explained above); namely, the nonindividuality of particles, on both the moderate and eliminativist construals of OSR. So, it could be argued, OSR is trying to have it both ways: on the one hand, they advise caution in reading the ontology directly off those formalisms, but, on the other hand, they do allow aspects of the formalism directly into their ontology, i.e., group structure as the basis for their non-individualist world-structure ontology. As a further counter-reply, the OSR theorist could claim once again, with some justification, that the non-individuals, holistic conception that informs their underlying ontology is, in fact, the common feature of all successful QM formalism so far, and possibly, QG formalisms as well, thus their choice of which element of the formalism to "reify" is both motivated and defensible. The ESR theorist will respond, of course, by recalling the long history of allegedly fundamental theories, and their ontologies, that have been overturned, and caution that the world-structure hypothesis may yet undergo the same fate. 
Accordingly, OSR is not without a plausible defense against the allegations put forward above. The main point of these last criticisms, to put it somewhat differently, is that by making structure ontic, the underdetermination of structure (of either the entity or formalism variety) should equate with an underdetermination of ontology, and thereby undercut the sort of hazy world-structure metaphysical interpretation actually offered by the OSR theorists. In contrast, the implications for the underlying ontology connected with the liberal brand of ESR has the advantage that it is, first, less metaphysically controversial than its OSR rival, and, second, that it is also a much more defensible scientific realist position (in opposition to anti-realism, that is). Since these structures need not reveal the "natures" the underlie our best theories, and the knowledge that they do provide is both fallible and revisable just as long as approximate structural continuity is preserved, ESR would seem to be the better scientific realist option. These considerations, finally, would seem to support many of the recent defenses offered on the behalf of ESR that raise analogous, if more general and less spacetime specific, themes (Morganti 2004, Saatsi 2008).

\section{Non-Realist Structuralisms and ESR.}

This section will compare and contrast various "non-realist" (i.e., neither realist nor anti-realist) structuralisms with the liberal brand of ESR put forward in section 1. As will be demonstrated, issues pertaining to the convergence of structure raise problems for these non-realist interpretations.

2.1. Early Structuralisms. Given its thin realist implications and obvious epistemic credentials, ESR bears a rough resemblance with the various conceptions of objectivity 
advanced by the early structuralists, as well as by both Friedman and van Fraassen.vii Since the objective/subjective dichotomy is a more forthrightly epistemological notion, it may have appealed to the early structuralists as a means of overcoming the thorny ontological issues that emerged in the new physics, with objectivity serving as a neutral position between the rival realist and anti-realist camps. As such, the transcendental idealism that characterizes the spacetime theorizing of Cassirer (via Marburg NeoKantianism), Weyl (via Husserlian phenomenology), and Eddington can be characterized as "non-realist", i.e., neither realist nor anti-realist, but is best deemed as simply opposed to subjective idealism or relativism: see, Ryckman 2005, who refers to Eddington's approach as "transcendental idealism shorn of "noumenalism"” (234), where "transcendental idealism" is "a metaphilosophical standpoint, beyond realism, idealism (or anti-realism)", (287). As is well-known, the structuralists employed the geometrical concept of an invariant, as a geometrical object preserved within a group of coordinate transformations, to capture their new understanding of the objectivity of knowledge. This approach drew inspiration from Klein's Erlangen program and Helmholtz, but received its most important impetus in the tensor formalism of GR. Overall, this branch of mathematics can be loosely described as the study of "what remains the same" (invariant) under different spatial perspectives - thereby demonstrating, in rough outline, how the objective features of geometry emerge from the subjective, with a structure (the transformation group) providing this objective knowledge (see, e.g., Weyl 1927, 116, Eddington 1939, 85-87). This group theoretic notion of an invariant, consequently, is of a far different kind than the so-called "invariant" of ESR, where the approximate continuity of successive theories is the intended meaning. Moreover, the various Kantian, 
Husserlian, etc., motivations that prompted the early structuralist theories are absent from contemporary ESR.

Cassirer called his system, a "universal invariant theory of experience", noting that the "procedure of the "transcendental philosophy' can be directly compared" to geometry: "Just as the geometrician selects for investigation those relations of a definite figure, which remain unchanged by certain transformations, so here the attempt is made to discover those universal elements of form, that persist through all change in the particular material content of experience" (1910/1921, 268-269). This hypothesis is first elaborated in Substance and Function, for Cassirer argues that the old conception of science/philosophy, as disclosing the world's underlying ontology (substances), must now be replaced by the more accurate estimation that regards science as obtaining a knowledge of the invariant mathematical interrelationships, framed by physical laws, (functions) manifest over the course of the empirical sciences. In the context of spacetime

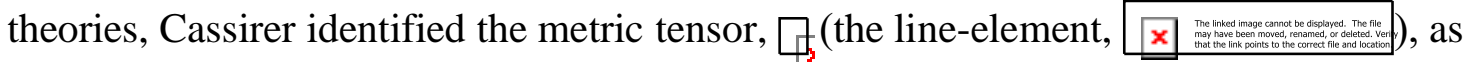
the geometric invariant corresponding to an a priori spatial component of knowledge (for the then current state of scientific theorizing, such that a deeper invariant may be revealed in the future; 1910/1921, 433). Because the coefficients ${ }_{x}$ of the metric tensor can encode any number of different geometries besides Euclidean, the flat geometry of Euclidean space in standard Newtonian theory can be seen as a particular instance of the larger metric group, but it is not an "approximation" of in some limiting process sense: it is an instance of $\square$. Only when the metric is coupled to the field equations, for both the Newtonian theory and GR, do you get the former as an approximation to GR in special cases (slow speeds and far away from massive bodies) via a limiting process. This 
distinction between regarding the geometric components, such as $\square$, alone or in the context of the larger theory is essential, and it remains unclear to what extent the early structuralists addressed this distinction. (More research is required here, although it is beyond the bounds of this essay). In Cassirer's case, for example, the retrospective search for functional invariants over the course of scientific theorizing, which could support either ESR or OSR, is only one facet of his philosophy of space. As briefly noted above, he also furnishes examples of spatial invariants that draw upon the notion of a transformation group so as to model the subjective/objective dichotomy in the manner of Weyl and Eddington (e.g., Cassirer 1979); and this facet of invariance might reflect the OSR approach more closely, since objectivity is attached to the whole group of transformations encoded by $\square$ (although the Kantian element in all of these earlier schemes are much more in league with ESR, see endnote 7).

2.2. Freidman's Relativized A Priori Structuralism. In several important essays, Michael Friedman has strived to resuscitate a conception of scientific knowledge that borrows elements from both Cassirer's neo-Kantianism and the notion of a revisable a priori adopted by some of the Logical Positivists (e.g., the early Reichenbach, and Carnap). As Friedman notes (2000, 116-118), Cassirer's understanding of scientific knowledge fits the Kantian classification of a purely "regulative" ideal, since the intellectual faculties alone determine the invariants of experience in a sort of abstract or reflective manner (such that this invariant content can only be established retrospectively, as the ESR theorist would also counsel). Friedman's theory of scientific knowledge, on the other hand, would like to preserve some facet of the "constitutive" process in cognition, which for Kant involved both the understanding and sensibility in a synthesis 
that renders comprehensible human sense experience (and is such that various synthetic a priori structures can be known to hold for all possible experience in advance, unlike the regulative process above). Friedman would like to counter both Quine's holism, which supposedly undermines all applications of an analytic/synthetic distinction, and the relativism implicit in Kuhn's theory of scientific revolutions.

In GR, Friedman's three-part division of theoretical structures can be described as follows: the mathematical structure is the semi-Riemannian manifold, which constitutes the spatiotemporal framework of the theory; the equivalence and light principles of GR form the coordinating principles that link the mathematical structures to the theory's empirical laws (respectively, the constancy of the velocity of light coordinates physical phenomena with the manifold's infinitesimally Minkowskian metric, and the equivalence principle coordinates the paths of "test particles" with the metric's geodesics); finally, there are the empirical laws, such as Einstein's field equations, which are only rendered empirically meaningful by the constitutive function of the mathematical structures and coordinating principles (and thus serve as a relativized a priori framework, contra Quine). Friedman contends that, through an analysis of the progressive historical development of these constitutive structures, "we can thus view the evolution of succeeding paradigms or frameworks as a convergent series, as it were, in which we successively refine our constitutive principles in the direction of ever greater generality and adequacy" (2001, 63), which thereby undermines Kuhnian relativism. While Friedman tends to avoid the issue, he does briefly comment on the relationship between his approach to rationality and scientific truth as it pertains to the realism/anti-realism quandary: toward the end of his Stanford lectures, he comments that his conception of scientific rationality "does not 
proceed on the basis of 'scientific realism'..." $(2001,118)$, and, in fact, "is consistent with ... 'anti-realist' conceptions of truth" (68n, original emphasis).

2.3. Convergence and its Realist Implications. Yet, even if the examination of science is transferred to the rarefied heights of rationality, there still remain important "realist" implications associated with this theory. In particular, Friedman simply assumes that the historical evolution of our best scientific theories will converge to a, presumably, finite set of relativized a priori structures (or, for Cassirer, mathematical/physical invariants, since Cassirer appears to make the same assumption). Friedman's hypothesis requires, first, "that earlier constitutive frameworks are exhibited as limiting cases, holding approximately in certain precisely defined special conditions, of later ones", and, second, that "the concepts and principles of later paradigms ... evolve continuously, by a series of natural transformations, from those of earlier ones" (63, emphasis added). Friedman's reference to earlier frameworks being limiting cases that approximate later frameworks is especially intriguing, since it is identical to Worrall's claims for SR (which would eventually be dubbed ESR). Friedman's estimate, accordingly, is that demanding a limiting approximation of successive frameworks is non-realist (neither realist nor antirealist), whereas Worrall reads this same demand as supporting (structural) realism. What are we to make of this predicament?: Is one person's realism another's non-realism?

While Friedman is certainly justified in regarding his view as non-realist, there would seem to be no justification for his assumption that the series of structures will continue to converge over the course of future scientific theorizing. For instance, it is conceivable that, within a particular science, an earlier theory (relativized a priori framework) may not be subsumed within a later theory (framework) as a special case of that later theory 
(i.e., the unity of science is violated). Therefore, as the defender of ESR will insist, the presumption of a converging series has an unmistakable, if slender, realist implication; where "converge" is defined as the earlier theories/frameworks being retained as limiting cases that approximate the latter theories/frameworks. That is, stipulating that the structures of theories converge is tantamount to endorsing the "no miracles" argument of ESR, since this argument, construed liberally, predicts this convergence, i.e., such that it is not merely an historical accident. Consequently, given that Friedman's neo-Kantianism and the liberal interpretation of ESR agree on this convergence prediction, Friedman appears to be in need of an explanation for his convergence prediction, whereas ESR does have one (we will address an objection to this critique of Friedman in section 3).

The anti-realist position, in contrast, would likely refrain from making any such convergence prediction, thus Friedman is not correct in maintaining that his theory is neutral between the realist and anti-realist positions. Unless the anti-realists would welcome a blurring of their thesis with ESR, invoking a future-directed convergence of scientific structures falls clearly on the realist side of the realism/anti-realism debate, since "empirical adequacy" would seem to be satisfied by any and all future patterns of scientific law formation as regards convergence or non-convergence. More specifically, the anti-realist philosophy seems consistent with the situation, described above, where future successful theories in a particular science fragment into a series of incommensurable "local" theories, such that each is confined to a restricted set of nonoverlapping phenomena, and where the combined empirical data of all of these theories are not subsumable within a larger theory: e.g., if no successor quantum gravity theory is ever found to incorporate GR and quantum theory; or, if Kepler's planetary laws and 
Galileo's free-fall law were never subsumed within Newton's gravitational theory.viii In fact, van Fraassen would seem to endorse this very possibility: "There cannot be in principle, but only as a historical accident, convergence to a single story about our world" (1991, 482); and, more recently, he has leveled this form of underdetermination argument specifically against structural realism (see van Fraassen 2008). Admissions of this sort would thus seem to undermine Friedman's attempt to declare the neutrality of his version of approximate continuity concerning the realism/anti-realism dichotomy (see section 3). Indeed, van Fraassen's interest in "empiricist structuralism" seems rooted in the controversy surrounding the relationship between the data models and phenomena (on the semantic view of theories), with all that it entails for the venerable observation/theory problem for empiricism, but which seems irrelevant for the convergence debate (see van Fraassen 2006, 2008: and, e.g., Bueno 1997, for a sophisticated treatment of structural empiricism that explores the relationship among these models).

\section{3. "Multiple Routes" and ESR.}

This section will explore further the role of convergence in scientific theories, as well as the related issues of constraints and compensatory adjustments, with the ultimate goal of developing the liberal version of ESR.

3.1. Convergence and Multiple Routes. In all fairness, Friedman does attempt to secure a rationale for his non-realism (neither realist nor anti-realist), and his arguments call to mind van Fraassen's allegations of the historical contingency of a "single story". Friedman, in effect, rejects the view that science is evolving towards a final, single conception of entities and/or structures, unlike scientific realism. There is "an essential 
element of convergence in the historical evolution of successive constitutive frameworks", he concludes, but insists that "this is explicitly not convergence to an entirely independent "reality" (however conceived) but rather convergence within the evolving sequence of constitutive frameworks itself" $(2001,118)$. Moreover, his "conception of scientific rationality does not even require that there be a uniquely correct sequence of convergent successor theories—-something that would certainly be required by any version of 'scientific realism"' $(2001,118)$. Friedman does admit "an element of 'internal' realism or what Kant called 'empirical realism”", since "once a given constitutive framework is already in place, there is a perfectly precise sense in which we can then speak of a 'matching' or 'correspondence' between a theory formulated within that framework and the empirical or phenomenal world" $(2001,118)$. In other words, Friedman only demands "that any reasonable route through [constitutive principles] be convergent", which implies there can be more than one route $(2001,68)$.

This is a very persuasive response, it should be noted: convergence need not aim at a final structure or entity, but can be simply interpreted as aiming at greater generality and empirical adequacy. Yet, the ESR supporter will point out that merely allowing for multiple constitutive frameworks does not free Friedman's theory from the necessity of acknowledging the constraints imposed on theoretical constructions, i.e., that there are "external" (real world) factors that constrain theory construction but which cannot be accurately tracked from within theories. These constraints are manifest in both (i) the fact that each framework in an evolving sequence appears as limiting approximation case of the later framework, and (ii), that not all frameworks are constructible. We have discussed (i) above, but (ii) requires more discussion. Put simply, Friedman's multiple 
routes hypothesis still faces the threat of subjective idealism (or relativism, solipsism), unless, of course, he admits that there are constraints in place to guarantee that not all constitutive frameworks, or all routes within a single framework, are candidates for his inter-theoretical version of realism. If any and all frameworks, and routes through a framework, are equally constructible and successful, then scientific theorizing would indeed appear to be a mere subjective creation, because frameworks that directly contradict one another on some specific prediction or theoretical commitment, say, $\square$ and $\square$, would be equally compatible with the same evidence, and thereby support a relativist construal of these frameworks. To borrow Laudan's distinction $(1996,42)$, Friedman's appeal to multiple routes would seem to constitute a case of nonuniqueness (more than one framework) rather than egalitarianism (all frameworks). But, even if the successful frameworks comprise a finite set, as opposed to just one, that still means that the majority of constructible frameworks are not successful-and thus an explanation is required to account for why some are successful and other are not. The realist would explain this failure of egalitarianism in terms of the constraints imposed by the world's ontology (as will be further discussed below). Of course, Friedman could counter that the needed constraints are supplied by the demand for greater communicative rationality and, especially, empirical adequacy at each step in a converging series of constitutive frameworks. Hence, Friedman is not without an explanation of constraints, although the manner by which these constraints operate to limit the egalitarian option would need to be explained in more detail than he has hitherto provided.ix The realist, on the other hand, has the benefit of a built-in explanation of the source of these constraints. 


\subsection{Constraints, Compensatory Adjustments, and the Two Requirements of Multiple}

Routes ESR. The multiple routes objection runs counter to a standard "entity" scientific realism, needless to say, since that thesis must defend the view that science is ultimately grounded upon a single, final ontological class of entities. But structural realism, and ESR in particular, need not endorse a corresponding final structure — and, in fact, there may be good reasons for endorsing the possibility of multiple routes, especially within the complex theoretical constructions of spacetime theories; e.g., formalism underdetermination. First, the liberal interpretation of ESR does not rule out the possibility that there can be more than one approximate limiting structure, or formalism, preserved over the course of scientific change; rather, it only holds that these structures are grounded upon the world's ontology. The construction of different spacetime theories from the same empirical basis is well-known in philosophy of physics circles, of course, as famously initiated by Poincaré's example (1905) of the alternative spatiotemporal interpretations of his disc-world inhabitants. Two theoretical constructions are consistent with the evidence obtained on the disc-world: (1) that the geometry is Euclidean but "universal forces" distort the measuring apparatus, or (2), the geometry is non-Euclidean and there are no such universal forces (see, e.g., Kosso 1997). The lesson for the structuralists, in addition to comprising an early instance of the underdetermination of theories, is that it is the "geometry + physics", $G+P$, that faces the tribunal of experience, and not simply the geometrical structure, $G$, alone. Spacetime structures, like all of the mathematical apparatus, must be coordinated to physical processes—and this leaves open the possibility that, in the limit of scientific theorizing, more than one $G+P$ combination may be consistent with the empirical evidence.x 
The multiple routes version of ESR has two requirements. First, the $G+P$ combinations must be nonunique rather than egalitarian, since, as above, admitting any and all such combinations would threaten realism/objectivism. In the Poincaré's example, not all aspects of the choice between (1) and (2) are conventional. If one decides to retain a flat space, then one must postulate strange forces that distort the measuring instruments. On the other hand, if one decides to directly accept these measurements, then one must conclude that the space is curved. Consequently, once a framework is conventionally chosen (respectively, that the space is flat or that the meter sticks provide accurate measurements), the conclusions that can be drawn about the world are, in large part, determined. Given a strong form of relativism/subjectivism, however, any geometry, and all assumptions about measuring instruments, should equally apply: for instance, option (3), that the geometry is Euclidean but no universal forces distort the apparatus. That this option is falsified by observational experience thus discloses the presence of "constraints" imposed on theory construction, constraints that seemingly come from "outside" the particular theoretical framework chosen by the scientist (since the framework itself cannot determine the nature and precise occasion of these constraints in advance). Put simply, by appealing to the underlying physical ontology, the scientific realist can thus provide a natural explanation for the manifest experience of theoretical constraints, whereas Friedman's rationality hypothesis must simply accept these constraints as merely brute facts of the conjunction of empirical adequacy and communicative rationality.

In Friedman (1983), the nonuniqueness of spacetime constructions is, in fact, openly acknowledged, for it comprises an important part of Friedman's case against metric 
conventionalism. In this earlier work, Friedman seems content to defend a general commitment to spacetime realism or absolutism, but not the ontological thesis, substantivalism - indeed, Friedman's reluctance to engage the deeper ontology debate in (1983), and his contention that various spacetime structures are preserved over the course spacetime theorizing (such that these structures receive repeated boosts in confirmation due to their unifying power) bears an uncanny resemblance with the ESR approach developed above: e.g., Friedman reckons that we should endorse "a realistic attitude toward the space-time structure $\times$ [the topological manifold $\square$ and the affine connection, $\square$, with the latter covering both the absolute and dynamical cases] . . . since the unifying power of this structure [i.e., $\mathrm{x}$ physics through GTR]" $(1983,261)$.xi Thus, given the basic similarity of Friedman (1983) and ESR, it is not surprising that one can locate a similar appeal to the nonuniqueness of spacetime constructions (as opposed to egalitarianism) in Friedman's discussion of the "compensatory adjustments" that are required when changing among empirically equivalent spacetime theories, i.e., our Poincaré-style geometric underdetermination. He concludes that the aspects of theories that actually contribute to empirical success cannot be merely changed or dropped without making "compensatory adjustments" to the rest of the theory $(1983,292)$ - hence, our cases (1) and (2) above furnish a simple instance of compensatory adjustments as applied to Poincaré's hypothetical disc world. Friedman's own example involving these same compensatory adjustments centers upon the decision to graft a non-Euclidean metric $\square_{x}$ to a Newtonian spacetime with a flat connection On the whole, this new theory, $\sqrt{x}$, will no longer be empirically equivalent to the earlier version of the theory, 
particles that comprise an expanding or shrinking body, due to $\square$, will fail to follow straight paths as determined by $\square$, consequently there will be no adequate Newtonian explanation for this fact in $\sqrt{x}$. To preserve the empirical equivalency of these theories, one must introduce a non-flat affine connection $\square$ compatible with $\square$. Therefore, one must change the laws of motion (i.e., the components of the affine connection) from $\mathbf{x}$

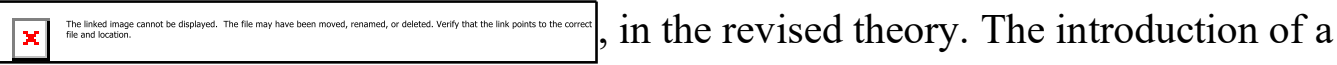
universal force, $\square$, that distorts the inertial paths, as in case (1), secures for this new theory, $x$, empirically equivalent predictions in keeping with Newtonianism.xii By vividly portraying the constraints imposed within various Newtonian spacetime constructions (whether our nonuniqueness clause on the construction of spacetime theories, and is thus in keeping with the multiple routes interpretation of ESR.

The second requirement for a multiple routes formulation of ESR is that each $G+P$ combination must, from its perspective, be interpreted as an invariant feature across all of the other combinations, $G^{\prime}+P^{\prime}$, that makeup the nonunique class of successful spacetime theories (so as to defeat the pessimistic meta-induction). That is, each $G+P$ combination must approximately contain all of the empirical data and predictions of all other $G^{\prime}+P^{\prime}$ combinations; and, returning to Poincaré's example, the "flat space plus forces" theory does indeed approximate empirically the "curved space without forces" theory.xiii (A similar restriction must be in place for Friedman's multiple routes hypothesis of scientific rationality.) 
In short, the multiple routes form of ESR simply concludes that there may remain a select handful of geometry plus physics combinations (and not just one) that save the phenomena. Friedman's analysis is thus informative for ESR since it discloses the importance of all the constitutive elements in scientific theories, both mathematical structures and coordinating principles, when searching for invariant structure. The multiple routes formulation of ESR does, however, allow for the nonuniqueness of multiple routes in a much more satisfactory manner than Friedman's (2001) rationality method. Finally, it should be noted that, in the limit of scientific theorizing, it may turn out that only one geometric structure, $G$, from within one $G+P$ pair, is consistent with the evidence and/or the scientific method's list of theoretical virtues, so ESR is not committed to a multiple routes outcome.

\section{Conclusion: Can OSR Take Multiple Routes?}

Before concluding, there is one more topic of interest to the structural realist concerning underdetermination and spacetime theories, namely, the possibility that OSR can adopt the "multiple routes" idea promulgated above. This possibility has been suggested by Brading and Skiles (2009) under the rubric of a "law-constitutive" account of objects: "what it is to be a physical object is to satisfy a certain system of physical laws", although "this is not to say that objects ontologically depend upon our theories about what those laws are, or even upon the laws themselves" (7; original emphasis). They caution that "there is no guarantee that this strategy will generate one unified kind of physical body: perhaps the bodies that serve as the subject matter of the laws when gravitation is included will turn out not to be identical to those that serve as the subject 
matter of the laws when electrical phenomena are at issue" (7).

While an intriguing idea, a good case can be made that this law-constitutive view more properly comes under the heading of ESR, rather than OSR. The example they provide, of a gravitational versus an electrical law-constitutive conception of ontology, invokes laws that are common to both, and which binds or constrain their application and scope; e.g., the conservation of mass-energy. While individually the application of these laws may not directly refer to the deeper conservation law (and GR, in fact, does not have a conservation law), their application will, nevertheless, be found to satisfy the conservation principle (as will our grand theories that unify these more specialized laws). This strongly suggests, therefore, that the separate constitutive laws are only capturing a mere facet of reality, and that they are constrained at a deeper level of reality by these other factors (e.g., the conservation law). Hence, it is difficult to interpret this situation as the ontic form of structural realism, since a deeper level of ontology constrains the application and scope of these separate laws (gravity and electrical). Given these circumstances, which are endemic to scientific theorizing in general, it seems more plausible to say that these separate constitutive laws are hence different epistemological perspectives of the world's single ontology, and not different ontologies altogether. The comment by Brading and Skiles that objects need not depend upon our theories would seem consistent with this last point.

On the other hand, a more radical form of this law-constitutive approach might actually favor the view that all aspects of ontology (objects) do depend upon these laws/theories— but this would be tantamount to the radical subjectivism worries previously examined, since there would be no explanation for, nor any basis for 
presupposing, the existence of constraints in theory construction unless one assumes the mere brute fact of these constraints. Indeed, this last option would place the lawconstitutive form of OSR in the same camp as Friedman and van Fraassen's non-realist hypotheses, as well as violate Saatsi's reasonable demand that any variant of "scientific realism" procure an explanation of past theoretical success (see endnote 1). All in all, what remains that is worthy of the SR label in this version of OSR? ESR, in comparison, at least offers the goal of a final explanation of past theoretical success, predicated, of course, on our experience of the approximate continuity of mathematical structure over the succession of past theories, through either a single route or multiple routes.

To sum up, the best option for the law-constitutive OSR theorist is to declare that ontology is itself hierarchical, with laws revealing perspectival aspects of the one ontology from various law-constitutive frameworks, but which thus posits (via the one, underlying and interconnected ontology) a vehicle for the constraints imposed on these higher-level frameworks. Yet, this view is ESR, since it accepts that our theories have not, and possibly may never, reveal the world's ontology in full detail. Construed in this fashion, the law-constitutive form of OSR could thus be viewed as a different, ontologyoriented "path" to the same conclusions reached via ESR. This last concession, moreover, helps to supports the main theme of this essay: specifically, that the underdetermination dilemma forces the various structural realist interpretations into adopting something that is suspiciously like ESR, or, as in the case of Friedman's non-realist neo-Kantian theory, mandates a similar convergence of approximate structure over theory change (which, as argued above, is the chief realist commitment of ESR).xiv 
i Saatsi (2008) has argued that a scientific realism requires more than mere approximate continuity, but should also explain the success of the earlier theory from the succeeding theory's vantage point. Yet, while quite correct as a final goal or heuristic of scientific realism, explanations of this sort would seem to require access to the ontology underlying these theories (or, at least, the succeeding theory's ontology), and the liberal form of ESR endorsed in this essay denies that this requirement is necessary to establish the success of ESR relative to its non-realist rivals - the reason being that the non-realist alternatives have no grounds for claiming a future directed convergence of approximate continuity over the course of science (see, sections 3 and 4).

ii See, e.g., French and Ladyman (2003), and Ladyman and Ross (2007) for more on the ontology of OSR. The characterization of this ontology as "holistic", along with many other descriptions, are outlined in Ladyman (2009). "World-Structure" is the term mentioned in Ladyman and Ross $(2007,158)$.

iii This is different from "metaphysical underdetermination" as mentioned in Ladyman (1998), which is generated by alternative realist interpretations of a single theory. Rather, entity underdetermination is the thesis that different theories, with different ontologies, will likely replace our currently successful theories. In essence, entity underdetermination is the ontological consequence of the pessimistic meta-induction.

iv Pooley $(2006,88)$ raises the formalism underdetermination issue, along with a number of metaphysical underdetermination objections (see, endnote 3). However, if the OSR theorist falls back upon their world-structure ontology, it is not clear that these metaphysical underdetermination cases can gain much traction against OSR. For instance, Pooley raises the specter that different interpretations of the measurement problem (e.g., de Broglie-Bohm versus other interpretations) lead to the underdetermination of the exact nature of the realist ontology, despite the use of the same mathematical formalism by these different interpretations (of the collapse of the wave function). Yet, since the same mathematical formalism is utilized, both collapse interpretations uphold the same world-structure ontology, with the difference lying in the mere details of how that ontology functions in the collapse case-and this is a much less troubling underdetermination than the entity or formalism underdetermination problems raised above, which do call into question the world-structure ontology itself.

v Bain (2009) strives to address the formalism underdetermination in Pooley (2006) (dubbed, "Jones underdetermination" by both authors). Bain's very promising analysis employs a category-theoretic approach in order to model a theory's dynamical structure (say, solutions of the field equations in GR) via the symplectic manifold that encodes the phase space of dynamically possible states. This conception may blunt the worries associated with formalism underdetermination, but it depends on a category theory framework, which many find problematic (Bain 2009, 17). Also, the many different constructions of GR occupy different symplectic manifolds (e.g., twistor models are different from tensor models with/without boundary conditions; 19), and so neither the "entity" nor the "metaphysical" underdetermination problems, as we have called them, 
have been diminished (see endnote 3). The OSR theorist can, of course, always claim that future empirical data might decide between these competing models, given their different dynamical structure - but the liberal brand of ESR theorist will interpret this same argument as support for their more cautious brand of SR (since the evidence may forever fail to distinguish these alternatives).

vi This understanding of ESR, in the context of spacetime theories, should be contrasted with the interpretation offered by Esfeld and Lam. Since they interpret ESR as a commitment to intrinsic properties, they conclude: "applied to the framework of the standard tensor representation of space-time, epistemic structural realism implies that the identity of the space-time points is constituted by their fundamental intrinsic properties, independently of the space-time structure - that is, independently of the metric" (35). In other words, ESR becomes Hartry Field's (1980) mathematical structure-reducing version of manifold substantivalism. But this does not follow at all, since ESR (on our liberal variety) remains agnostic as regards the underlying ontology, and thus the manifold points may have an ontological status that is either intrinsic, or, like OSR, is either derived from, or "on a par" with, the metric. In short, Esfeld and Lam's reading of ESR foists a commitment to a particular mathematical ontology (of points) when its only commitment is to some underlying ontology.

vii There is, of course, a great deal of subjective license involved in attempting to translate early twentieth century neo-Kantian theories into the modern OSR/ESR dichotomy, but, I would argue, their approach clearly falls on the epistemological side of that dichotomy, and not the ontological. Given the predominant emphasis on conceptual categories and their like in shaping our experience of the world, drawing purely ontological lessons, apart from these epistemic components, seems quite problematic. Friedman's discussion of the goals of Cassirer's Substance to Function makes this point clear: "[I]n accordance with the 'critical' theory of knowledge, ... convergence, on this view, does not take place towards a mind- or theory-independent 'reality' of ultimate substantial 'things'.... 'Reality', on this view, is simply the purely ideal limit or endpoint towards which the sequence of [theoretical] structures is mathematically converging — or, to put it another way, it is simply the series itself, taken as a whole" (Friedman 2005, 75).

viii A number of objections can be raised at this point. First, the neo-Kantian might claim that a mathematical invariant can always be found that links the totality of empirical data, thus rejecting the possibility just noted (although, as a counter-reply, this would be difficult to prove). Second, the anti-realist may insist that empirical adequacy dictates that all empirical data must be subsumed by more general theories (if not, empirical adequacy would be sacrificed). However, as argued previously, this demand would render antirealism practically indistinguishable from ESR, since it basically admits that scientific theories must converge.

ix Can Friedman's neo-Kantian insist, as does the realist, that the "world" provides the needed constraints to rule out the egalitarian option? While the "world" obviously plays a major role in scientific theorizing for Friedman, to claim that it explains convergence 
would clash with his insistence that his view is consistent with anti-realism. As explained above, van Fraassen's anti-realism holds that convergence is contingent; so, if Friedman really accepts that his view is consistent with anti-realism, then he would need to add that convergence may also be a mere accident. I believe that Friedman's past history of supporting realism, along with his demand for convergence (explored above), makes it unlikely that he would regard convergence as merely a contingent accident.

x A famous counter-argument against structural realism, originally introduced by $\mathrm{M}$. Newman, should be briefly addressed at this point, although we will only explore a version of the argument adapted for the semantic view of theories: if a mathematical structure represents the world by standing in an isomorphic relationship with the world's structure, then one can undermine the uniqueness of this representation by introducing another world domain of the same cardinality, and carving out a structure that is also isomorphic to the mathematical structure in this new domain. As argued by French and Saatsi (2004), however, this problem can be overcome by including interpretations of the mathematical structure's theoretical variables, so that they refer to a particular group of properties and relations. This reply to the Newman problem thus parallels Poincaré's insight, i.e., that structures are always linked to the world via coordinating principlesand hence our $G+P$ approach to structure naturally includes French's and Saatsi's defense. Friedman has himself contributed to the Newman argument, see, Demopoulos and Friedman (1985).

xi That is, Friedman (1983) appears to endorse a realist commitment to spacetime

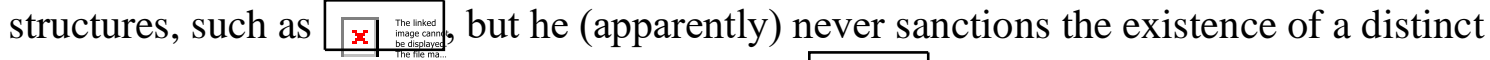

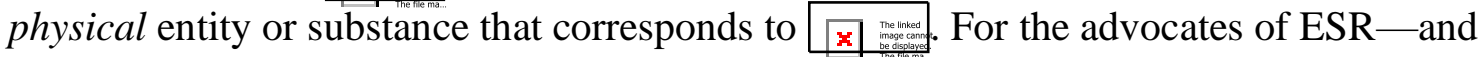
possibly Friedman (1983) - the substantival/relational dispute in ontology (i.e., whether spacetime is a substance or relation among substances) is a separate issue from the reality (=objectivity) of the spacetime structures themselves (in conjunction with the coordinating principles). In other words, both the substantivalist and relationist hypotheses are compatible with the minimalist ontological implications of our liberal brand of ESR (i.e., the convergence of spacetime theories and the manifest constraints/nonuniqueness of spacetime constructions), although both will obviously differ on the deeper ontological foundations.

xii Friedman (1983, 297-299). That is, the force $\square$ now explains why the particles that comprise an expanding or shrinking body, due to $\square_{x}$ deviate from straight-line trajectories as the body moves, apparently inertially, throughout various regions of space. Here, the $x$ are the flat components of $\square_{x}$, and the $x$ are the non-flat components of

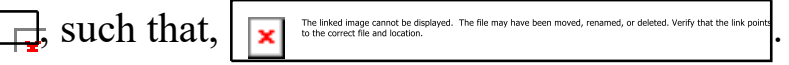

xiii An anti-realist or OSR realist may object, at this point, that the multiple routes form of ESR is tantamount to an equivalence of structure at the empirical level only, which is simply insufficient to qualify as a realist theory. This objection, however, fails to take into account the fact that our version of ESR is, indeed, a very liberal brand of realism (see endnote 1 and sections 2 and 3): given the two requirements of the type of ESR 
theory advocated in this essay-nonuniqueness, and that each nonunique $G+P$ combination must be an invariant feature across all of the other nonunique combinations, $G^{\prime}+P^{\prime}$ - the "realism" in this theory is manifest in two important ways, (1) via predictions on future theoretical constructions (which runs counter to anti-realism, or is at least quite difficult for the anti-realist to explain), namely, that theories will converge; and (2) that there will exist constraints on the theoretical constructions (thus eliminating the egalitarian option along with its relativist implications for epistemology and ontology). Finally, it should be noted that a famous species of underdetermination concerning spacetime theories is consistent with this Poincaré-inspired exegesis, as well as with the brand of ESR defended in this essay: namely, the topological underdetermination manifest by observationally indistinguishable spacetime theories, as explored by Malament (1977) and Glymour (1977).

xiv An earlier version of this paper was presented at the Philosophy of Science Association 2006 conference, Vancouver, B.C., Canada. I would like to thank the participants for their helpful discussions, especially Oliver Pooley, Carl Hoefer, Steven Weinstein, Vincent Lam, Nick Huggett, and Bas van Fraassen, and two anonymous referees from the European Journal for Philosophy of Science. 


\section{REFERENCES}

Bain, J. (2006). "Spacetime Structuralism", in The Ontology of Spacetime, vol. 1, ed. by D. Dieks, Amsterdam: Elsevier, 37-66.

Bain, J. (2009). "Motivating Structural Realist Interpretations of Spacetime", Metaphysics of Science (Melbourne, July 2-5, 2009), Philsci-archive.

Bueno, O. (1997). "Empirical Adequacy: A Partial Structures Approach", Studies in History and Philosophy of Science, 28, 585-610.

Brading, K. and Landry, E. (2004). "A Minimal Construal of Scientific Structuralism", Philosophy of Science, 73, 571-581

Brading, K. and Skiles, A. (2009). "Underdetermination as a Path to Ontic Structural Realism”, Minnesota Center for the Philosophy of Science Colloquium, 2009.

Butterfield, J. and Isham, C. (2001). "Spacetime and the Philosophical Challenge of Quantum Gravity", in Physics meets Philosophy at the Planck Scale, ed. by C. Callender and N. Huggett. Cambridge: Cambridge University Press, 33-89.

Cassirer, E. (1910/1921, [1952]). Substance and Function and Einstein's Theory of Relativity, trans. by W. C. Swabey and M. C. Swabey, New York: Dover.

Cassirer, E. (1979, [1945]). "Reflections on the Concept of Group and the Theory of Perception", in Symbol, Myth, and Culture, ed. by D. P. Verene. New Haven: Yale University Press.

Demopolous, W. and Friedman, M. (1985). "Critical Notice: Bertrand Russell's The Analysis of Matter: Its Historical Context and Contemporary Interest", Philosophy of Science, 52, 621-639.

Dorato, M. (2000). "Substantivalism, Relationism, and Structural Spacetime Realism", Foundations of Physics, 30, 1605-1628.

Eddington, A. (1939, [1958]). The Philosophy of Physical Science. Ann Arbor: University of Michigan Press.

Esfeld, M. and Lam, V. (2008). "Moderate Structural Realism about Space-time", Synthese, 160, 27-46.

Field, H. (1980). Science Without Numbers (Princeton: Princeton University Press).

French, S. (1989). "Identity and Individuality in Classical and Quantum Physics", Australasian Journal of Philosophy, 67, 432-446. 
French, S. and Ladyman, J. (2003). "Remodelling Structural Realism: Quantum Physics and the Metaphysics of Structure", Synthese, 136, 31-56.

French, S. and Saatsi, J. (2004). "Realism about Structure: The Semantic View and Nonlinguistic Representations", Philosophy of Science, 73, 548-559.

French, S. and Rickles, D. (2006). "Quantum Gravity Meets Structuralism: Interweaving Relations in the Foundations of Physics", in The Structural Foundations of Quantum Gravity, ed. by Rickles, D., French, S., and Saatsi, J. Oxford: Oxford University Press, 139.

Friedman, M. (1983). Foundations of Space-Time Theories. Princeton: Princeton University Press.

Friedman, M. (2005). "Ernst Cassirer and The Philosophy of Science", in Continental Philosophy of Science, ed. G. Gutting. Oxford: Blackwell, 71-83.

Friedman, M (2000). A Parting of the Ways: Carnap, Cassirer, and Heidegger. Chicago: Open Court.

Friedman, M. (2001). Dynamics of Reason. Stanford: CSLI Publications.

Friedman, M. (2005). "Ernst Cassirer and the Philosophy of Science", in Continental Philosophy of Science, ed. by G. Gutting, London: Blackwell, 71-84.

Glymour, C. (1977). "Indistinguishable Space-Times and the Fundamental Group", in Foundations of Space-Time Theories, Minnesota Studies in the Philosophy of Science, vol. 8., ed. By J. Earman, C. Glymour, \& J. Stachel. Minneapolis: University of Minnesota Press, 50-60.

Hedrich, R. (2009). "Quantum Gravity: Motivations and Alternatives", philsciarchive.pitt.edu/archive/00004820.

Hoefer, C. (1996). "The Metaphysics of Space-Time Substantivalism", Journal of Philosophy, 93, 5-27.

Kosso, P. (1997) Appearance and Reality. Oxford: Oxford University Press.

Ladyman, J. (1998). "What is Structural Realism?", Studies in the History and Philosophy of Science, 29, 409-424.

Ladyman, J. and Ross, D. (2007). Every Thing Must Go: Metaphysics Naturalized. Oxford: Oxford University Press.

Ladyman, J. (2009). "Structural Realism", Stanford Encyclopedia of Philosophy, http://plato.stanford.edu/entries/structural-realism/ 
Laudan, L. (1996). Beyond Positivism and Relativism. Boulder: Westview Press.

Malament, D. (1977). "Observationally Indistinguishable Space-Times", in Foundations of Space-Time Theories, Minnesota Studies in the Philosophy of Science, vol. 8., ed. by J. Earman, C. Glymour, \& J. Stachel. Minneapolis: University of Minnesota Press, 61-80.

Morganti, M. (2004). "On the Preferability of Epistemic Structural Realism”, Synthese, $142,81-107$.

Poincaré, H. (1905, [1952]). Science and Hypothesis, trans. by W. J. Greenstreet, New York: Dover.

Pooley, O. (2006). "Points, Particles, and Structural Realism", in The Structural Foundations of Quantum Gravity, ed. by Rickles, D., French, S., and Saatsi, J. Oxford: Oxford University Press, 83-120.

Psillos, S. (2004). "The Structure, the Whole Structure and Nothing but the Structure?", Philosophy of Science, 73, 560-570.

Ryckman, T. (2005). The Reign of Relativity. Oxford: Oxford University Press.

Saatsi, J. (2008). “Whence Ontological Structural Realism”, EPSA 2007 Proceedings.

Saunders, S. (1993). "To what physics corresponds", in Correspondence, Invariance and Heuristics: Essays in Honour Of Heinz Post, ed. by S. French and H. Kamminga. Dordrecht: Kluwer, 295-325.

Slowik, E. (2005). "Spacetime, Ontology, and Structural Realism", International Studies in the Philosophy of Science, 19, 147-166.

van Fraassen, B. (1991). Quantum Mechanics: An Empiricist View. Oxford: Oxford university Press.

Weyl, H. (1927, [1949]). Philosophy of Mathematical and Natural Science. Princeton: Princeton University Press.

Worrall, J. (1989), “Structural Realism: The Best of Both Worlds?”, Dialectica, 43, 99124. 